\title{
Design and Implementation of Smartphone Rapid Triage APP in Emergency Medical Rescue System
}

\author{
Chen $\mathrm{Yu}^{1, \mathrm{a}}$,Zheng Zhi-hao ${ }^{2, \mathrm{~b}}$,Ren Jia-shun ${ }^{1, \mathrm{c}}$,Chen \\ Shang-kun ${ }^{3, \mathrm{~d}}$,Xiang $\mathrm{Yu}{ }^{1, \mathrm{e}}$ \\ ${ }^{1}$ The Third Military Medical University, XinQiao \\ Hospital,ChongqingChina \\ ${ }^{2}$ Chongqing Institute of Green and Intelligent Technology, Chongqing \\ China \\ ${ }^{3}$ Chongqing Beijiou Software Co.,Ltd., Chongqing China \\ achenyu_63@163.com, ${ }^{b} z h i h a o @ c i g i t . a c . c n,{ }^{c}$ renjs028@163.com, \\ dcsk83@qq.com, ${ }^{e}$ xiangyu0719@163.com
}

\begin{abstract}
Objective: To explore the new methodto improve the efficiency of mass wounded rapid triage in emergency rescue system. Methods: Develop the Smartphone rapid triage client (APP)in emergency medical rescue system. Results: Smartphone triage APP costs 2/3 shorter time than the writing paper mode, and shorten the time of handwritten electronic records of 1/3 than PDA mode. Conclusion: Intelligent Smartphone occupies a large quantity, APP's develop fast, APP triage is simply operated, raises mass wounded triage efficiency.

Keywords: Triage, Medical rescue, APP, Smartphone
\end{abstract}

\section{Introduction}

APP (Application) stands for the Smartphone application software or Smartphone client. In the era of rapid development of mobile Internet, mobile APP's have been more and more popular, and they have become the mainstream of the mobile internet [1]. At present, APP development market is making a spurt of progress, Smartphoneapplications are not only widely used in Products marketing, information release, financial payment and other aspects, but the application of which in the medical field has also been unfolding, especially in emergency medical rescue system, masses of woundedSmartphonerapid triage 
APP'sare taking advantages[2].

\section{Batch of Wounded Triage Demand}

At the time ofwars, natural disasters and public health events explosions, the wounded population increasessharply inshort time internal, while the medical rescue resources of the scene is not sufficient [3]. At this time, triage of the wounded contributes greatly to rational utilization of the limited manpower, triage and classification;alsoit is effective for a large number of wounded to be disposed with the fastest speed [4]. Thewounded classification method (Simple Triage And Rapid Treatment, START) is adopted in many countries and regions, namely, the action check, respiratory examination, inspection, examination cycle level of consciousness, and according to the classification principle onthe critical and severe, mild and death, it identifies priority treatment, no-treatment and deferred treatmentperson, so as to maximize the survival rate of the wounded, and to reduce the degree of disability as far as possible,and then, transportthe wounded safely and timely to medical institutions for further treatment.

Therefore, the main task of triage is to make a preliminary classification based on the wounded injury, and immediately start treatment with orderly arrangement [5]. As a result, to decide quickly and record briefly as well as make clear treatment is required to the triage.

\section{SmartphoneTriage APP Design Idea}

In the medical rescue practices in the past, most of the rescue teamsused paper triage mode to record the wounded information, injury part and conditions; only a handful of rescue teams used PDA triage equipment, handwritten notes were recorded about the wounded information, injury part and conditions, and transferred to the medical rescue workstation [6]. But there still exits many problems like:lost and damage to paper records, high error rate of PDA handwritten records, un- informational system application.

The key of the Smartphone triage APP is to improve the triage and make the implementation more effective, with the focus of the wounded information 
management, to ensure the efficiency of the wounded rescuing, the development process of Smartphone triage APP is shown in figure 1. With the premise of occupying a large quantity, low cost and simple operation, easy to learn and use in the Smartphone, rapid triage applied in mobile APP's in emergency medical rescue of the wounded in batches, can greatly optimize and standardize the triage process, it will also improve the triage mode and efficiency andstrive for the successful rescue time.

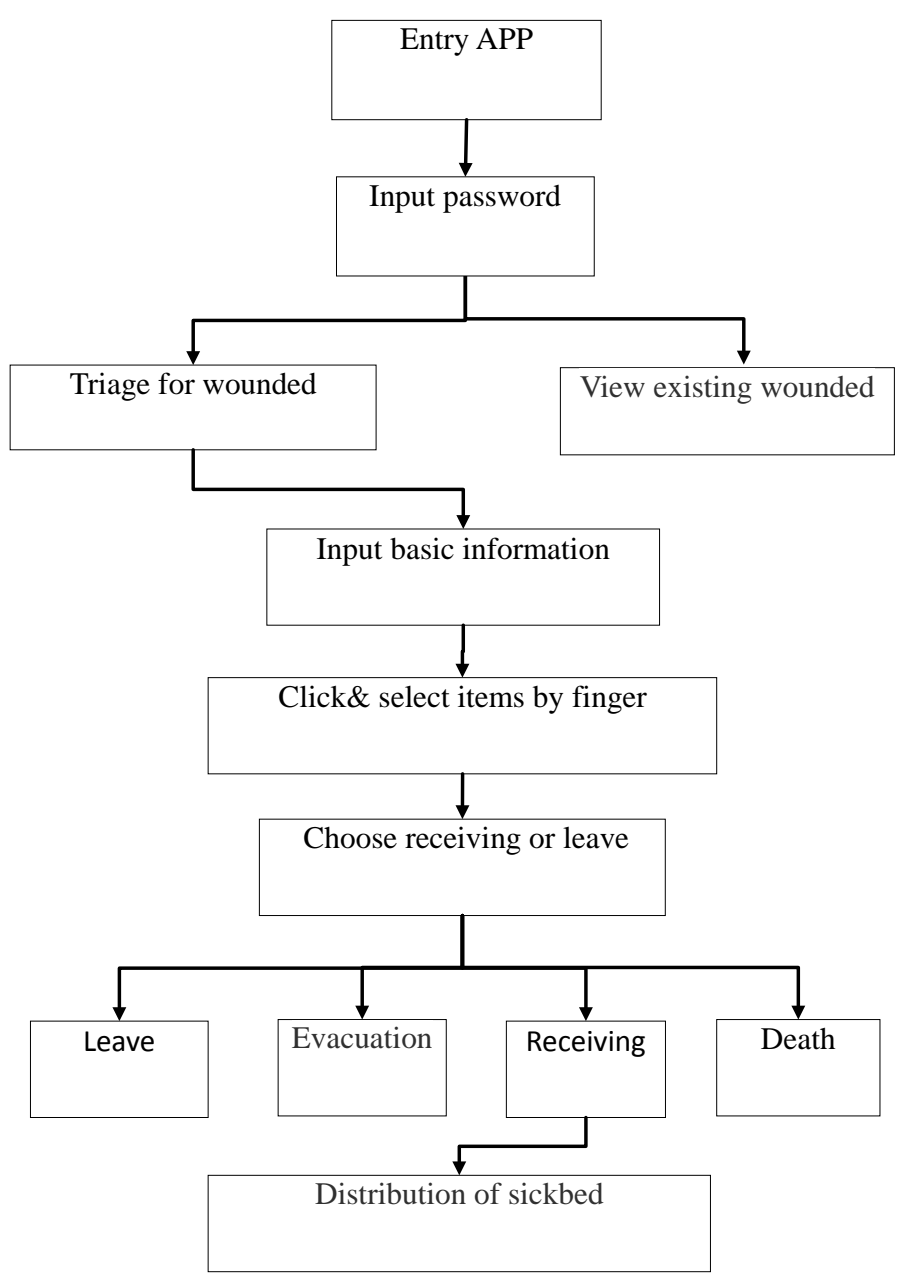

Fig.1 The frame of Smartphone triage APP 


\section{Smartphone APP Design}

Under WiFi environment, with the platform of Huawei P6 intelligent Smartphone and T440 notebook, the Web and RESTfull interface protocolis designed and developed tobe the intelligent Smartphone triage APP, the sketch structure is schematically shown in figure 2.

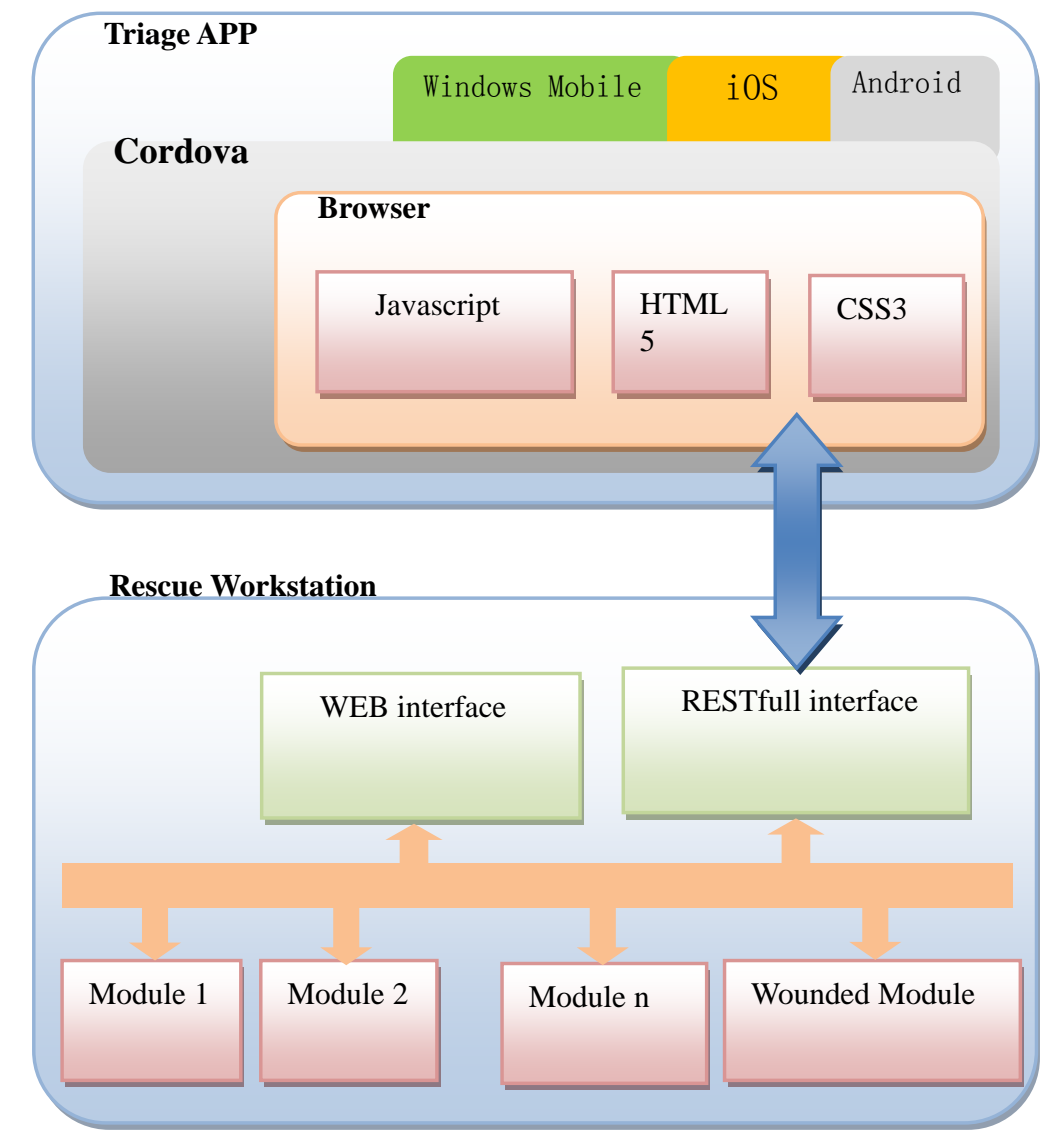

Fig. 2 The sketchof Smartphone triage APP

The Smartphone triage APP is not only supported in close the WiFi environment, but could also be used in traditional mobile networks. The implementation case selects WIFI local area network environment, afterthe operation of logging in the APP, the user should open Webpage medical rescue workstation, and click triage elements according to the medical personnel triage 
decision, then, the wounded should be immediately transferred to the medical rescue workstation. The APP design of the icon, click page is shown in figure 3.

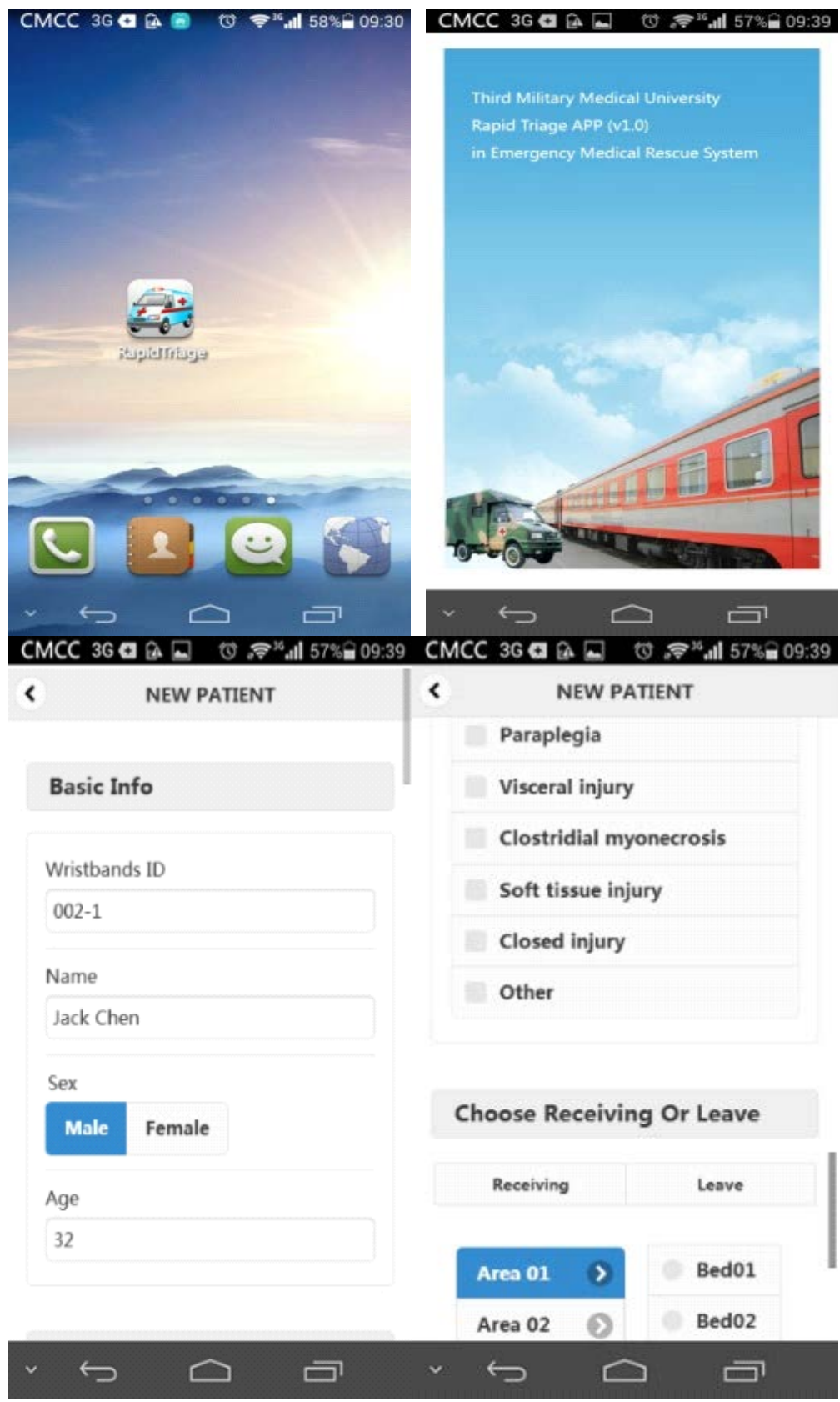

Fig. 3 The screens of Smartphone triage APP 
In order to make the APP software to adapt to a variety of intelligent Smartphone platforms, the developer uses a browser package open-source technology development based on a variety of system operation (Cordova). With the help of embedded browser method to run across systems, the workload and technical barriers to the native development of different systems is reduced, and the main power of development is focused on the service logic and data exchange. This APP is combined of HTML5, CSS3, APP Javascript, Ajax, Java and other technologies, using RESTFull mode to communicate with the server data, and JQuery, JQueryMobi to be used to set up the APP UI interface; the server adopts PHP, Mysql API way to interact with the APP data.

The Smartphone triage APP's section codes of display program is as follows:

$<$ div class="ui-field-contain">

<fieldset data-role="controlgroup" data-type="horizontal"> $<$ legend $>$ shangshi $</$ legend $>$

$<$ ?php

\$html = ";

if(!empty(\$_shangshi) $)\{$

foreach(\$_shangshi as \$item) \{

\$code $=$ \$item['item_code'];

\$name $=$ \$item['item_name'];

\$html .= '<input type="radio" name="shangshi"

id="shangshi-'.\$code."' value="'.\$code."'><label

for="shangshi-'.\$code."'>'.\$name.'</label>';

\}\}

echo \$html;?>

$<$ /fieldset $>$

$</ \operatorname{div}>$

The Smartphone APP and the rescue workstation network trial in the command and decision system of whole continuous treatmentchainshows that: the Smartphone triage APP has the following advantages: login simply, 
click-select fast, transmitefficiently, as well as easy to use. In the simulation test of batch of wounded rapid triage, thisSmartphone triage APP costs 2/3 shorter time than the writing paper mode, and improves $1 / 3$ by time than the PDA electronic record mode.

\section{Conclusion}

The Smartphone per capita amount is large, easy to carry, and is already deeply rooted in people's lives, it has wide application prospects in the field of medicine [7]. With the process of identity card module and RFID read-write module chip becoming more and more popular, IC card is used to input wounded basic information, read and write the wounded classification in emergency rescue inspection, so as to further improve the efficiency of mass wounded triage, and savemore precious time for successful treatment of the wounded.

\section{Acknowledgements}

This work was sponsored by the projects of state science and technology plans ( Project No.2012BAI21B04 ) .The corresponding author: Ren Jia-shun,Email: renjs028@163.com.

\section{References}

[1]S.C. Tong, H. Zhang, M.M. Zhai, et al. The design of intelligent mobile phone APP auxiliary digital patient[J]. Chinese Medical Equipment Journal, 2014, 7:58-59. (Chinese)

[2]F. Xiao, X.Q. Guo, Z.D. Huang, et al. The second generation ID card stored value card for medical treatment to realize the design of self-service and charging system[J]. Military Medical Journal of South China, 2012, 26(1): 66-68. (Chinese)

[3]Q.R. Niu. The direction of construction of mobile medical APP[J]. China Digital Medicine, 2014, 4:26-28. (Chinese)

[4]R. Luo. USA mobile medical APP: novel to the mainstream of the road[J]. 
China Medical News, 2014, 11. (Chinese)

[5]Z.D. Huang, X.Q. Guo, W. Zhang, et al. The application and implementation of HIS platform based on the stored value medical card system[J]. Chinese Medical Equipment Journal, 2010, 31(11):67-68. (Chinese)

[6]G.H. Fan, Y.X. Zhao, G.Q. Yao, et al. Design and construction of digital hospital outpatient system[J]. Military Medical Journal of South China, 2013, 27(5):344-346. (Chinese)

[7]G.Y. Zhang. Medical APP will change the medical model[J]. China Medical News, 2014, 19. (Chinese) 